\title{
Histological Evaluation of Hydroxyapatite Granules with and without Platelet-Rich Plasma versus an Autologous Bone Graft Comparative study of biomaterials used for spinal fusion in a New Zealand white rabbit model \\ Zamzuri Zakaria, ${ }^{1}$ Che N. Z. C. Seman, ${ }^{1}$ Zunariah Buyong, ${ }^{2}$ "Mohd A. Sharifudin, ${ }^{1}$ Ahmad H. Zulkifly, ${ }^{1}$ Kamarul A. Khalid ${ }^{1,3}$

$$
\begin{aligned}
& \text { تقوبك النسجيات لحبيبات الهيدروكسي آباتيت في وجود أو عدم وجود بالزما } \\
& \text { غنية بالصفائح الدموية مقابل طُّم عظمي ذاتي إني }
\end{aligned}
$$

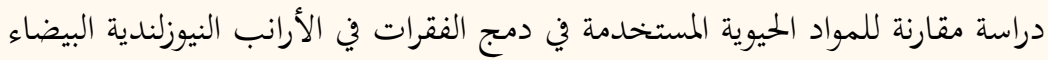

زمزوري زكريا، شي نور زارد اشي سيمان، زانريا بيونغ، محمد عارف شرف الدين، أحمد حافظ ذو الكفل، قمرول آرفن خالد

ABSTRACT: Objectives: Hydroxyapatite (HA) has osteoconductive properties and is widely used as a bone graft substitute. Platelet-rich plasma (PRP) is an autologous product with osteoinductive effects. Hypothetically, a combination of both would augment the bone formation effect of HA and widen its application in spinal fusion surgeries. This study aimed to compare new bone formation with HA granules alone and in combination with PRP versus an autologous bone graft during a lumbar intertransverse process spinal fusion. Methods: A total of 16 adult New Zealand white rabbits underwent single-level bilateral intertransverse process fusion at the L5-L6 vertebrae. One side of the spine received either HA granules alone or a combination of HA granules and PRP, while the contralateral side received an autologous bone graft. Four animals each from the HA group and the HA plus PRP group versus the autograft group were assessed either at six or 16 weeks by undecalcified histology and histomorphometry. The mean percentage of new bone areas over the corresponding fusion masses were compared between groups. Results: No significant difference in new bone formation was observed between the HA and HA plus PRP groups at six or 16 weeks. The autograft group had significantly more new bone formation at six and 16 weeks $(P=0.004$ and $<0.001$, respectively). Conclusion: An autologous bone graft remains superior to HA granules, with or without PRP. HA granules demonstrated an excellent osteoconductive scaffold but had poor biodegradability. While PRP enhances the properties of HA granules, these biomaterials do not have a synergistic effect.

Keywords: Autografting; Hydroxyapatite; Lumbar Vertebrae; Platelet-Rich Plasma; Spinal Fusion.

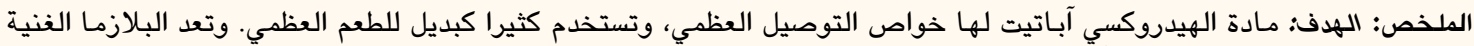

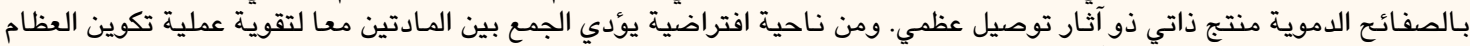

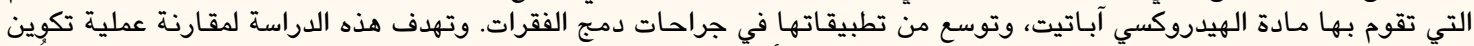

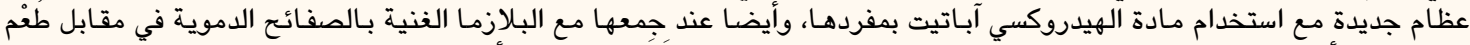

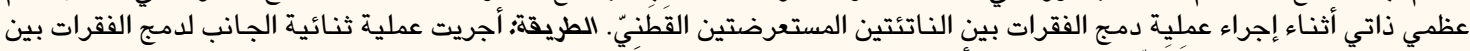

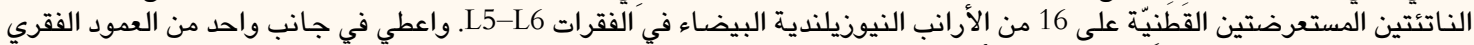

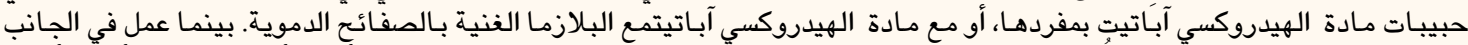

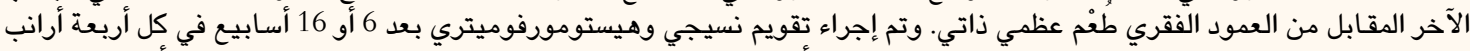

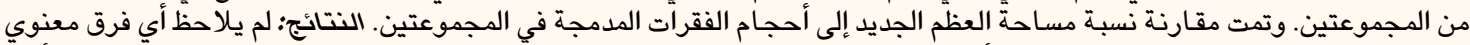

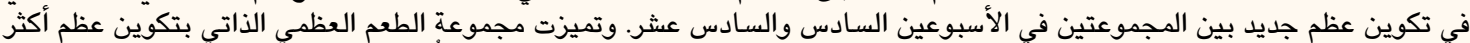

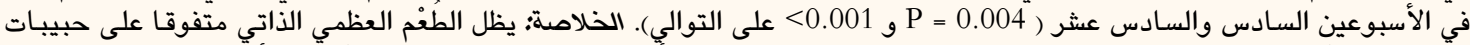

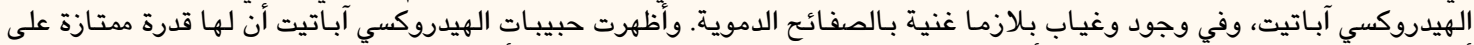

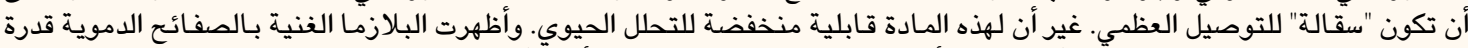

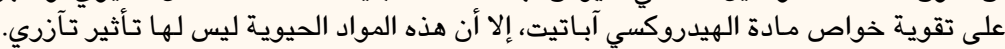

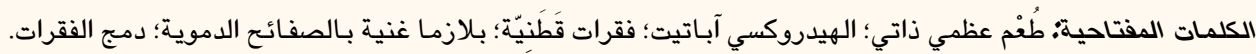




\section{ADVANCES IN KNOWLEDGE}

Calcium phosphate-based hydroxyapatite (HA) granules can be used as bone substitutes in non-weight bearing areas such as in a lumbar intertransverse process spinal fusion.

HA granules have excellent osteoconductive properties but lack biodegradability.

Autologous bone grafts result in significantly more new bone formation in comparison to HA granules, with or without platelet-rich plasma (PRP).

\section{Application to Patient Care}

The results of this study showed that HA granules and a combination of HA granules and PRP are potentially good bone substitutes in spinal fusion procedures; however, autologous bone grafts result in significantly more new bone formation.

\section{B} ONE GRAFTS ARE USED IN VARIOUS CLINICAL fields. ${ }^{1,2}$ Commonly, bone grafts are harvested from the patient's own bones during surgery. However, serious complications related to the harvesting procedure can arise, such as bleeding, infection and chronic pain at the donor site, as well as increased costs and operating time. ${ }^{1,3,4}$ As a result, the demand for bone graft substitutes has led to multidisciplinary research designed to develop new biomaterial technology.

In Malaysia, researchers from a collaborative project have successfully manufactured and commercialised a synthetic bone graft substitute known as GranuMas $^{\circledR}$ (GranuLab, Kuala Lumpur, Malaysia); this product is made of locally produced calcium phosphate-based hydroxyapatite (HA) granules fabricated from limestone. ${ }^{5}$ Khadijah et al. tested this bone graft substitute on New Zealand white rabbits with non-critical tibial metaphyseal defects; undecalcified bone histology showed new bone formation two weeks after implantation and the defect was completely bridged by new bone at $4-6$ weeks. ${ }^{6}$ As demonstrated in the animal model, HA granules have shown good evidence of bone formation in the weightbearing areas; however, there is currently no scientific evidence that this product is suitable for application in non-weight-bearing areas, such as in lumbar spinal fusion.

In lumbar spinal fusion surgeries, an autologous bone graft is still considered the gold standard, as it possesses all of the properties necessary for bone regeneration, such as osteoconductivity, osteoinductivity and the presence of osteogenic cells. ${ }^{3}$ In comparison, HA granules are solely osteoconductive due to their similarity to the mineral components of bone tissue. ${ }^{7,8}$ Despite this, combining HA granules with growth factors-such as platelet-derived growth factor, transforming growth factor and vascular endothelial growth factor-can augment bone formation. ${ }^{9}$ The main purpose of this study was therefore to compare new bone formation using an undecalcified histology technique in a lumbar intertransverse process spinal fusion with HA alone and a combination of $\mathrm{HA}$ with platelet-rich plasma (PRP) versus an autograft control.

\section{Methods}

A total of 16 New Zealand white rabbits between 10-19 months old and 2.25-3.40 kg were used in this study. The animals were randomly divided into two groups of eight rabbits each to form a HA group and a HA plus PRP group. Two assessment periods were chosen to show differences in the fusion healing and remodelling changes as a previous study indicated histological evidence of fusion-healing changes at 16 weeks. ${ }^{10}$ As such, each group was further subdivided into two groups of four rabbits according to the time of observations (at six or 16 weeks, respectively).

Assumptions for the mean percentage of bone area in subgroups were made according to sample size calculations, since a previous study reporting a similar method of comparison using new bone formation in intertransverse process spinal fusions was performed at 12 -week intervals. ${ }^{10}$ The sample size was calculated using PASS software, Version 2008 (NCSS LLC, Kaysville, Utah, USA), to measure the difference of the mean percentage of bone area in subgroups with $80 \%$ power and a significance level of $<0.05$. In a oneway analysis of variance (ANOVA) test, the means of three samples of four individuals each were compared. The total sample of 16 subjects achieved $91 \%$ power to detect differences among the means versus the alternative of equal means using an F-test with a $<0.05$ significance level. The size of the variation in the means was represented by their standard deviation (24.94). The common standard deviation within a group was assumed to be 20.00 [Table 1].

All of the animals underwent a bilateral intertransverse process spinal fusion between the L5 and L6 vertebrae using a previously described lateral approach technique. ${ }^{11}$ A single orthopaedic surgeon with previous experience in animal studies performed the surgery and a trained laboratory technician administrated the anaesthesia. Synthetic GranuMas ${ }^{\circledR}$ HA granules (GranuLab) between 1,000-2,500 $\mu \mathrm{m}$ with a tap density of $0.524 \mathrm{~g} / \mathrm{mL}$ and $\sim 48 \%$ porosity were used. The platelet-rich plasma (PRP) was obtained from the rabbits' peripheral blood using a gravitational platelet sequestration technique with a table-top centrifuge machine. ${ }^{12}$ The processing 
Table 1: Sample size calculations for assumptions of the mean percentage of bone areas

\begin{tabular}{lcccccccc} 
Power & Average & $\mathbf{k}$ & Total & Alpha & Beta & $\begin{array}{c}\text { SD of } \\
\text { means }\end{array}$ & SD & Effect size \\
\hline 0.90861 & 4.00 & 3 & 12 & 0.05000 & 0.09139 & 24.94 & 20.00 & 1.2472
\end{tabular}

SD = standard deviation

was done under sterile conditions. The HA granules and platelet gel were prepared prior to implantation following the decortication of the transverse processes. The HA granules were mixed with the platelet gel in a Petri dish before being implanted into the fusion bed. One side of the spine was then implanted with either HA granules alone (the HA group) or HA mixed with PRP (the HA plus PRP group). The contralateral site of the spine acted as a control and received an iliac crest bone graft (the autograft group). The same rabbit was used in both one experimental group and the control group so as to eliminate the confounder of physiological variability between individual rabbits.

Subsequently, the rabbits were euthanised at either six or 16 weeks post-implantation. Their spines were harvested from the L4 to L7 vertebrae en bloc with the adjacent paravertebral muscles. A hard tissue band cutting and microgrinding system (Exakt Advanced Technologies, Norderstedt, Germany) was used to produce undecalcified tissue sections. Retrieved specimens were fixed in $10 \%$ neutral buffered formalin, dehydrated in a series of concentrations (70\%, 90\% and $100 \%$ ) of ethanol and then cleared in chloroform. The specimens were then infiltrated in various concentrations $(10 \%, 30 \%, 50 \%, 70 \%, 90 \%$ and $100 \%)$ of hydroxyethyl methacrylate (Technovit ${ }^{\circledR} 7200$, Heraeus Kulzer, Hanau, Germany) before being embedded in pure hydroxyethyl methacrylate (Technovit ${ }^{\circledR} 7200$, Heraeus Kulzer). The tissue sections were then stained using the Masson-Goldner trichrome staining method and analysed using a transmitted light microscope (Olympus ${ }^{\circledR}$ BX61, Olympus Corp., Tokyo, Japan).

The total area of fusion mass was defined as the area spanning from the upper border of the transverse process of L5 vertebra (TP5) superiorly, the lower border of the transverse process of L6 vertebra inferiorly, the intertransverse membrane ventrally and the ventral border of paraspinal muscles. New bone area was defined as an area with mineralised bone, marrow within the mineralised area and osteoids. Unremodelled or native transverse processes, marrow within the transverse processes, cartilaginous tissue, soft tissue stroma, HA granules and the remnants of the autograft were excluded from the measurement of new bone.

The total fusion and new bone areas were measured in pixels using Photoshop CS4 Extended, Version 11.0 (Adobe Systems Inc., San Jose, California, USA). Examples of the calculation of fusion mass, measured total fusion and new bone areas are shown in Figure 1.

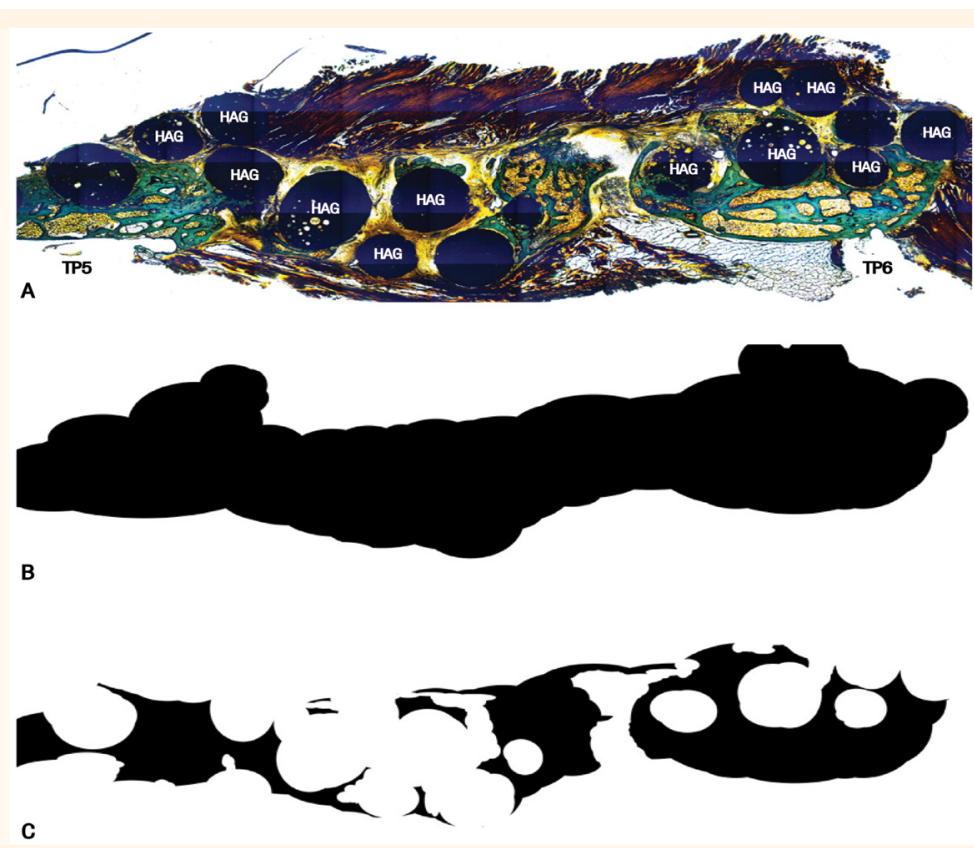

Figure 1: Schematic diagrams showing examples of the quantification of areas of (A) full thickness fusion mass, (B) total fusion mass and $(\mathbf{C})$ new bone in a New Zealand white rabbit model receiving an autograft and either hydroxyapatite granules or hydroxyapatite granules plus platelet-rich plasma.

$H A G=$ hydroxyapatite granules; TPS = transverse process of the $L 5$ vertebra; TP6 = transverse process of the L6 vertebra. 


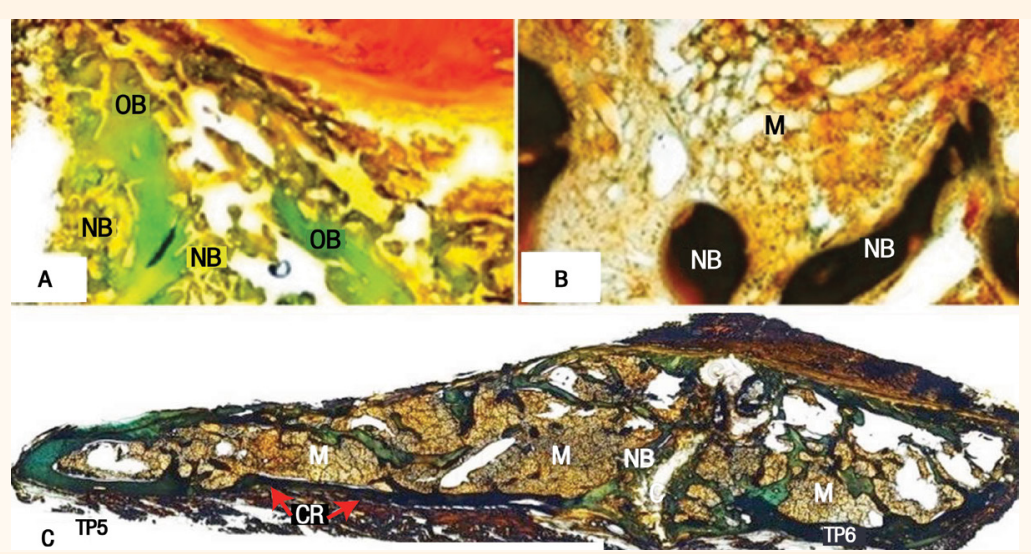

Figure 2: Photomicrographs of Masson-Goldner trichrome-stained sagittal sections at either (A \& B) six or (C) 16 weeks post-implantation showing the full thickness fusion mass of a New Zealand white rabbit receiving an autograft. A: Old bone (OB) can be seen in direct contact with the new bone (NB) trabeculae at x100 magnification. B: Islets of remodelled bone trabeculae and marrow as well as NB, cartilage and remnants of the autograft or OB are seen across the fusion mass at x200 magnification. C: Note the completely remodelled NB and almost complete cortical rim (arrows) bridging the bone between the transverse processes at $\times 40$ magnification. There is also visible loss of the normal anatomical structure of the transverse process of the L6 vertebra (TP6) and a common shared marrow cavity with the fusion mass. The soft tissue gap near the TP6 is filled with soft tissue stroma, various stages of endochondral bone cartilage formation and minimal remnants of $\mathrm{OB}$. $O B=$ old bone; $M=$ marrow; $N B=$ new bone; $C R=$ cortical rim; TP5 = transverse process of the $L 5$ vertebra; TP6 = transverse process of the L6 vertebra.

The percentage of new bone area was calculated from the total number of pixels filled with new bone over the total number of pixels in the fusion mass area. The mean percentage of new bone over the total fusion mass area in the autograft, HA and HA plus PRP groups at different periods were analysed using the Statistical Package for the Social Sciences (SPSS), Version 16 for Macintosh (IBM Corp., Chicago, Illinois, USA). The percentage of new bone was then compared statistically using the one-way ANOVA and Tukey's and Dunnett's post hoc tests. A P value of $<0.050$ was considered statistically significant.

This study was approved by the International Islamic University Malaysia Research Ethics Committee, International Islamic University Malaysia, Kuala Lumpur, Malaysia (\#IIUM/305/14/1).

\section{Results}

At six weeks post-implantation, the fusion mass in the autograft group was well defined and easily differentiated from the surrounding paraspinal muscle, which was stained brick red. Evidence of remodelled woven bone was observed in the fusion mass near the middle of TP5. Immature new bone trabeculae or woven bones were seen in the peripheral zones around the transverse processes and adjacent to both decorticated sides of the transverse processes. In the central zone, the fusion mass was made up of immature new bone trabeculae, bone marrow, remnants of old bones (either autografted or grafted bones), cartilage and soft tissue stroma.
In the autograft group, the remnants of old bones at six weeks were in close contact and integrated well with the islands of newly formed bone. Wellorganised and regularly arranged collagen fibres and osteocyte lacunae characterised the remnants of the old bones. The lacunae appeared empty and contained no osteoids. In this section, the green mineralised old bone appeared brighter than the new bone (woven or newly remodelled bone). In the same way, the undecorticated side of the transverse processes also displayed a green colour similar in intensity to the old bones [Figure 2A].

Areas of endochondral bone formation in the autograft group at six weeks post-implantation were characterised by a different chondroblast morphology. The chondroblasts in the small lacunae nearest to the stromal tissue were evenly spaced in the matrix of the reserve zone. Adjacent to this, the chondroblasts were stacked up in columns in the proliferative zone. Next to this zone, the lacunae were hypertrophied in the maturation zone. Subsequently, the hyaline cartilages were calcified and resorbed by osteoclasts in the resorption zone. Osteoblasts were attached to the calcified hyaline cartilage and secreted osteoids. Osteoids were observed scattered in the calcification zones and the adjacent woven bone. New bone trabeculae in these areas appeared less dense compared to the newly formed woven bone. In between bone trabeculae, cells of various sizes-suggestive of bone marrow-were observed [Figure 2B]. Evidence of adipose cells was noted in the marrow during this observation period. 

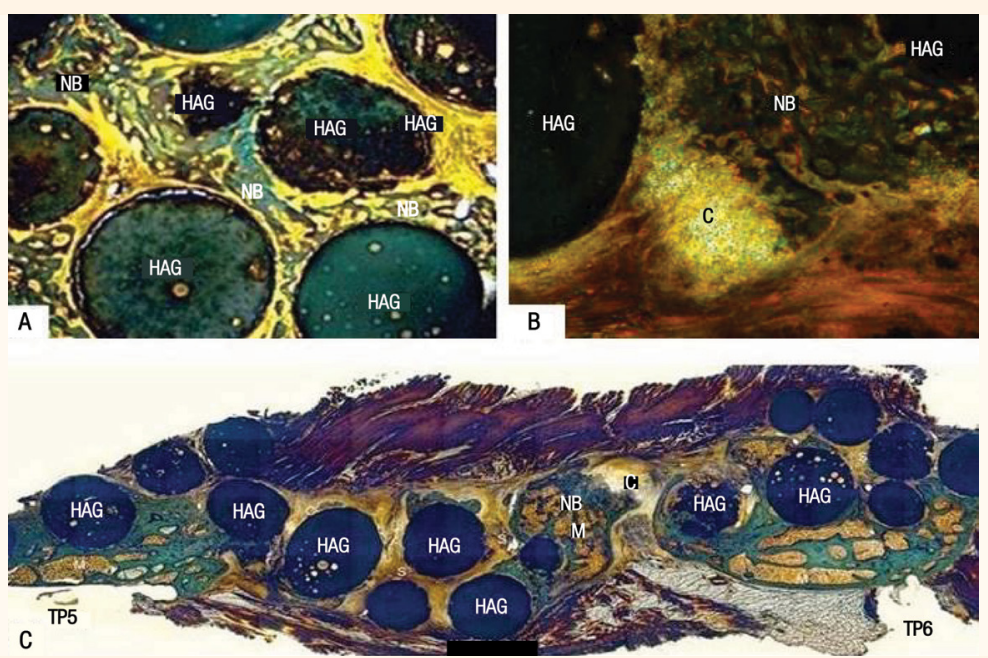

Figure 3: Photomicrographs of Masson-Goldner trichrome-stained sagittal sections at either (A \& B) six or (C) 16 weeks post-implantation showing the full thickness fusion mass of a New Zealand white rabbit receiving hydroxyapatite (HA) granules. A: The HA granules can be seen in direct contact with the new bone (NB) trabeculae at x10 magnification. B: Stromal tissue and endochondral bone formation are observed in proximity to the HA granules at x20 magnification. C: There is evidence of NB remodelled with bone marrow formation at $x 4$ magnification. Stromal tissue and many loci of on-going endochondral bone formation can be seen in close proximity to the HA granules. Evidence of a cartilaginous gap can be observed at the lower third of the fusion mass and there is no obvious cortical rim formation.

$N B=$ new bone; $H A G=$ hydroxyapatite granules; $C=$ cartilaginous zone; $M=$ marrow; $T P 5=$ transverse process of the $L 5$ vertebra; TP6 = transverse process of the L6 vertebra.

In the autograft group, minimal grafted bone was observed at 16 weeks post-implantation. The remnants consisted mainly of cortical bone. A striking feature seen at this stage was the almost complete remodelling of the fusion mass, characterised by the presence of a cortical rim encasing cancellous bone and marrow within it. Under higher magnification, various sizes of cells were seen, suggestive of the multicellular content of the bone marrow. These cells were separated from each other by empty spaces. Remnants of transverse processes underwent total remodelling as part of the fusion mass [Figure $2 \mathrm{C}$ ].

Six weeks post-implantation, new bone trabeculae were observed in the peripheral zones of the HA group. In the central zone, new bone trabeculae in between the HA granules almost bridged the transverse processes. Areas of soft tissue stroma and adjacent endochondral bone formation were also seen. There was extensive direct bonding between the new bone and the HA granules [Figure 3A]. Interestingly, new bone trabeculae had formed extensively on the surface of the HA granules in the fusion mass; however, no obvious new bone trabeculae were observed on the surfaces of the HA granules in contact with the margins of the fusion mass, neither at its junction with the paraspinal muscles nor the intertransverse membrane. At higher magnification, bone ingrowth was observed in some of the peripheral pores of the HA granules. No obvious remodelling was seen in the fusion mass at this time [Figure 3B].
In the HA group, scattered groups of stromal tissue and endochondral bone formation were seen in between the HA granules in the central region, similar to those observed in the autograft group. At 16 weeks post-implantation, new bone remodelled with the formation of bone marrow was observed [Figure 3C]. Scattered stromal tissues and on-going endochondral bone formation in the central zone were observed. No obvious evidence of early cortical rim formation was noted, unlike in the autograft group during the same observation period.

Generally, the HA granules in the HA plus PRP group were well defined and contained within the intended fusion site. In addition, fusion masses were better formed and a larger number of HA granules were observed on tissue sections in comparison to the HA group. Six weeks post-implantation, new bone trabeculae were seen only near the decorticated transverse processes (i.e. in the peripheral zone). In the central zone, a greater number of HA granules were contained in the fusion mass compared to the HA group. The spaces in between the HA granules were filled with either soft tissue stroma or dense material [Figure 4A]. The dense material was more prominent in spaces further from the decorticated transverse processes. Similar dense material was not found in either the autograft or the HA groups. The soft tissue stroma in the HA plus PRP group was composed of a few blood vessels and spindle-shaped fibroblast-like cells [Figure 4B]. Some stromal tissue 


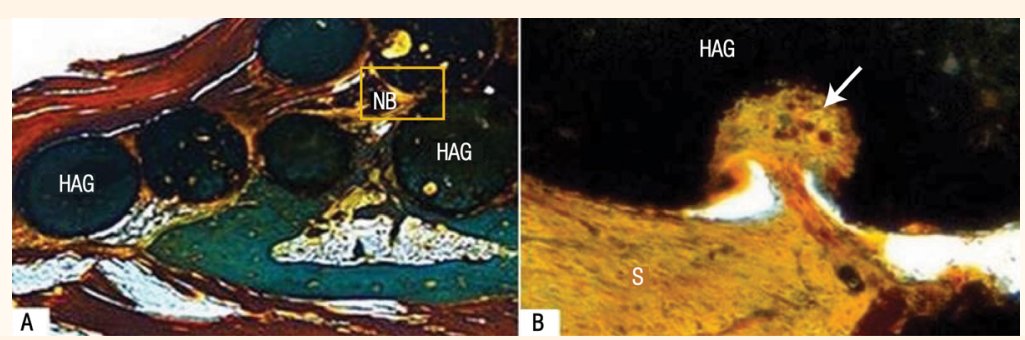

Figure 4: Photomicrographs of Masson-Goldner trichrome-stained sagittal sections at six weeks post-implantation showing the full thickness fusion mass of a New Zealand white rabbit receiving hydroxyapatite (HA) granules plus plateletrich plasma (PRP). A: Minimal new bone trabeculae are observable at x100 magnification and are adjacent to the transverse processes only. B: By enlarging an area of Figure 4A (yellow box) by x 20 magnification, a cavity in the periphery of the HA granule can be seen filled with stromal tissue and a multinucleated cell.

$H A G=$ hydroxyapatite granules; $S=$ stromal tissue.

was also seen in the cavities at the periphery of the granule. In one of the cavities, a multinucleated cell was discernible; however, whether this cavity was caused by osteoclastic activity or not could not be determined. No obvious new bone trabeculae were seen in close proximity.

In the HA plus PRP group, new bone areas were found only near the transverse processes at 16 weeks post-implantation. In contrast to the six-week samples, areas with endochondral bone formation were located in the peripheral zone. In the central zone, the fusion mass was packed with HA granules. Unlike the granules in the HA group, the granules in the HA plus PRP group were closer together. The spaces in between the granules were filled with dense material and some stromal tissue, similar in appearance to those observed at six weeks. No obvious new bone growth was seen in the central zone in any of the samples.

The mean percentage of new bone area in the autograft group was significantly higher compared to the HA and HA plus PRP groups at six weeks postimplantation $(65.5 \%$ versus $33.1 \%$ and $9.8 \%$, respectively; $P=0.004)$. At 16 weeks, the mean percentage of new bone area formed in the autograft group was $86.4 \%$ compared to $33.5 \%$ in the HA group and only
$20.3 \%$ in the HA plus PRP group $(P<0.001)$ [Table 2]. Dunnett's post hoc test showed no statistically significant difference between the HA and HA plus PRP groups $(P=0.154)$. A post hoc test revealed that significant differences existed only between the autograft versus HA groups and the autograft versus HA plus PRP groups ( $P<0.001$ each) and no significant differences were observed between mean bone percentages in the HA versus HA plus PRP groups $(P=0.487)$.

\section{Discussion}

A lumbar intertransverse fusion is commonly performed on patients with lumbar instability to regenerate bone tissue at sites previously occupied by muscle. Recently, HA has been used as a substitute for autologous bone grafting and there have been no detailed reports of intertransverse fusion failure. ${ }^{13,14}$ Calcium phosphate-based HA is well known for its osteoconductive properties; however, studies have revealed that graft materials with osteoconductive properties alone are inadequate to generate new bone at this site..$^{15-17}$ In its natural form, PRP contains growth factors and other cytokines; ${ }^{18,19}$ these growth

Table 2: Mean percentage of new bone growth between groups receiving an autograft and either hydroxyapatite or hydroxyapatite plus platelet-rich plasma at six and 16 weeks post-implantation in a New Zealand white rabbit model $(\mathrm{N}=16)$

\section{Six weeks post-implantation \\ n \\ Mean percentage \pm SD}

\section{6 weeks post-implantation}

$F(2,10)=9.808$

0.004
Mean percentage \pm SD

\section{Group}

\section{Autograft}

HA

F statistic

Pvalue

$$
\begin{gathered}
65.5 \pm 28.4 \\
33.1 \pm 9.0 \\
9.8 \pm 9.0
\end{gathered}
$$

$\begin{array}{lc}8 & 65.5 \pm 28.4 \\ 4 & 33.1 \pm 9.0 \\ 4 & 9.8 \pm 9.0\end{array}$

$86.4 \pm 12.0$

$33.5 \pm 12.6$

$20.3 \pm 9.1$

$S D=$ standard deviation $H A=$ hydroxyapatite $P R P=$ platelet -rich plasma 
factors have been shown to play an important role in promoting bone healing. ${ }^{20}$ Thus, hypothetically, the combination of HA granules and PRP would enhance the effect of HA granules on bone formation.

In the current study, there was new bone formation at both ends of the fusion mass near the decorticated transverse processes in the HA group at six weeks. No intervening cartilage was seen in close proximity to this area. Areas of cartilage formation were observed in between the granules in the central zone. At 16 weeks, new bone was seen bridging the transverse processes. However, unlike in the autograft group, there was an absence of cortical remodelling and single marrow cavities in the HA group. This presumably occurred because most of the area in the fusion mass consisted of HA granules. In the HA plus PRP group, specimens showed almost the same results as those of the HA group. New bone formation was limited at both ends of the fusion mass near the decorticated transverse processes. Both of the transverse processes appeared to be healed instead of demonstrating the active bone outgrowth seen in both the autograft and HA groups. Moreover, no intervening cartilage was seen in close proximity to this area. The spaces between the granules in the central zone were mainly occupied by connective tissue stroma and no areas of cartilage formation were observed. One sample even demonstrated mature tissue fibrosis at 16 weeks. In contrast, no tissue fibrosis was observed in samples from the autograft or HA groups. Furthermore, there was no statistical difference between the HA and the HA plus PRP groups; this may be due to the small sample size of the current study as the power to demonstrate a difference between the groups was small.

Although HA demonstrates good osteoconductivity, the mean percentage of bone area among the HA group in the current study was significantly lower; this may be because the HA granules were minimally resorbed even at 16 weeks post-implantation and they occupied a large volume of the fusion mass. A similar explanation can be applied to the HA plus PRP group as well. Nevertheless, the results of this study indicate that HA granules and HA plus PRP may potentially be used as a good bone graft substitute for spinal fusion. Other studies in the literature are in agreement with these findings.,21,22 The porous characteristics of HA potentially affect the histological outcomes of posterolateral lumbar fusion; Motomiya et al. found that the highly interconnected porous structure of HA was a promising environment for spinal fusion in a rabbit model in terms of producing fusion masses with higher cellular viability. ${ }^{23}$ In addition, Bansal et al. reported that $\mathrm{HA}$ in combination with $\beta$-tricalcium phosphate and bone marrow aspirate was a safe option for spinal fusion and had comparable effectiveness to an autologous bone graft; the major advantage of this option is the avoidance of donor site morbidity. ${ }^{2}$

Based on the results obtained from the current study, it is recommended that future research on HA granules or HA granules plus PRP focuses on the use of these materials in combination with spinal fusion instrumentation in larger-sized animal models. In spinal surgery, higher fusion rates are achieved when instrumentation is used. ${ }^{24}$ One of the limitations of the current study was the absence of macroscopic, radiographical and biomechanical analyses of the fusion areas.

\section{Conclusion}

Autologous bone grafts remain superior to the implantation of HA granules or HA granules plus PRP in terms of new bone formation in intertransverse process spinal fusion. However, HA granules-either in combination with PRP or on their own-can be used as alternative bone substitutes to support new bone growth.

\section{CONFLICT OF INTEREST}

The authors declare no conflicts of interest.

\section{FUNDING}

This study was supported by a research grant from the International Islamic University Malaysia (\#FRGS 0207-69).

\section{References}

1. Conway JD. Autograft and nonunions: Morbidity with intramedullary bone graft versus iliac crest bone graft. Orthop Clin North Am 2010; 41:75-84. doi: 10.1016/j.ocl.2009.07.006.

2. Bansal S, Chauhan V, Sharma S, Maheshwari R, Juyal A, Raghuvanshi S. Evaluation of hydroxyapatite and betatricalcium phosphate mixed with bone marrow aspirate as a bone graft substitute for posterolateral spinal fusion. Indian J Orthop 2009; 43:234-9. doi: 10.4103/0019-5413.49387.

3. David R, Folman Y, Pikarsky I, Leitner Y, Catz A, Gepstein R. Harvesting bone graft from the posterior iliac crest by less traumatic, midline approach. J Spinal Disord Tech 2003; 16:27-30.doi: 10.1097/00024720-200302000-00005.

4. Robertson PA, Wray AC. Natural history of posterior iliac crest bone graft donation for spinal surgery: A prospective analysis of morbidity. Spine (Phila Pa 1976) 2001; 26:1473-6. doi: 10.1097/00007632-200107010-00018.

5. Fazan F, Besar I, Osman A, Samsudin AR, Khalid KA. Successful commercialisation of locally fabricated bioceramics for clinical applications. Med J Malaysia 2008; 63:49-50.

6. Khadijah K, Mashita M, Saidu MF, Fazilah F, Khalid KA. Histological evaluation of the early bone response to hydroxyapatite (HA) implanted in rabbit tibia. Med J Malaysia 2004; 59:123-4. 
7. Damien CJ, Parsons JR. Bone graft and bone graft substitutes: A review of current technology and applications. J Appl Biomater 1991; 2:187-208. doi: 10.1002/jab.770020307.

8. Saikia KC, Bhattacharya TD, Bhuyan SK, Talukdar DJ, Saikia SP, Jitesh P. Calcium phosphate ceramics as bone graft substitutes in filling bone tumor defects. Indian J Orthop 2008; 42:169-72. doi: 10.4103/0019-5413.39588.

9. Anitua E, Andia I, Ardanza B, Nurden P, Nurden AT. Autologous platelets as a source of proteins for healing and tissue regeneration. Thromb Haemost 2004; 9:4-15. doi: 10.1160/ TH03-07-0440.

10. Boden SD, Schimandle JH, Hutton WC. An experimental lumbar intertransverse process spinal fusion model: Radiographic, histologic, and biomechanical healing characteristics. Spine (Phila Pa 1976) 1995; 20:412-20.

11. Zunariah B, Zakaria Z, Zarida CN, Rosnani AJ. Posterolateral intertransverse lumbar arthrodesis in the New Zealand white rabbit model: The illustration of an alternative surgical approach. Int Med J Malaysia 2012; 11:19-22.

12. Aghaloo TL, Moy PK, Freymiller EG. Evaluation of plateletrich plasma in combination with anorganic bovine bone in the rabbit cranium: A pilot study. Int J Oral Maxillofac Implants 2004; 19:59-65.

13. Gupta MC, Maitra S. Bone grafts and bone morphogenetic proteins in spine fusion. Cell Tissue Bank 2002; 3:255-67. doi: 10.1023/A:1024605128411.

14. Spivak JM, Hasharoni A. Use of hydroxyapatite in spine surgery. Eur Spine J 2001; 10:S197-204. doi: 10.1007/s005860100286.

15. Magit DP, Maak T, Trioano N, Raphael B, Hamouria Q Polzhofer G, et al. Healos/recombinant human growth and differentiation factor-5 induces posterolateral lumbar fusion in a New Zealand white rabbit model. Spine (Phila Pa 1976) 2006; 31:2180-8. doi: 10.1097/01.brs.0000232823.82106.0a

16. Glazer PA, Spencer UM, Alkalay RN, Schwardt J. In vivo evaluation of calcium sulfate as a bone graft substitute for lumbar spinal fusion. Spine J 2001; 1:395-401. doi: 10.1016/ S1529-9430(01)00108-5.
17. Bozic KJ, Glazer PA, Zurakowski D, Simon BJ, Lipson SJ, Hayes WC. In vivo evaluation of coralline hydroxyapatite and direct current electrical stimulation in lumbar spinal fusion. Spine (Phila Pa 1976) 1999; 24:2127-33. doi: 10.1097/00007632199910150-00012.

18. Eppley BL, Woodell JE, Higgins J. Platelet quantification and growth factor analysis from platelet-rich plasma: Implications for wound healing. Plast Reconstr Surg 2004; 114:1502-8. doi: 10.1097/01.PRS.0000138251.07040.51

19. Weibrich G, Kleis WK, Hafner G, Hitzler WE. Growth factor levels in platelet-rich plasma and correlations with donor age, sex and platelet count. J Craniomaxillofac Surg 2002; 30:97-102. doi: $10.1054 /$ jcms.2002.0285.

20. Marx RE, Carlson ER, Eichstaedt RM, Schimmele SR, Strauss JE, Georgeff KR. Platelet-rich plasma: Growth factor enhancement for bone grafts. Oral Surg Oral Med Oral Pathol Oral Radiol Endod 1998; 85:638-46. doi: 10.1016/S1079-2104(98)90029-4.

21. Siebrecht MA, De Rooij PP, Arm DM, Olsson ML, Aspenberg P. Platelet concentrate increases bone ingrowth into porous hydroxyapatite. Orthopedics 2002; 25:169-72. doi: 10.3928/ 0147-7447-20020201-22.

22. El Backly RM, Zaky SH, Canciani B, Saad MM, Eweida AM, Brun F, et al. Platelet rich plasma enhances osteoconductive properties of a hydroxyapatite- $\beta$-tricalcium phosphate scaffold (Skelite) for late healing of critical size rabbit calvarial defects. J Craniomaxillofac Surg 2014; 42:e70-9. doi: 10.1016/j. jcms.2013.06.012.

23. Motomiya M, Ito M, Takahata M, Kadoya K, Irie K, Abumi K, et al. Effect of hydroxyapatite porous characteristics on healing outcomes in rabbit posterolateral spinal fusion model. Eur Spine J 2007; 16:2215-24. doi: 10.1007/s00586-007-0501-0.

24. Bridwell KH, Sedgewick TA, O’Brien MF, Lenke LG, Baldus C. The role of fusion and instrumentation in the treatment of degenerative spondylolisthesis with spinal stenosis. J Spinal Disord 1993; 6:461-72. doi: 10.1097/00002517-19930606000001 